\title{
Blind Reception of Multicarrier DS-CDMA Using Antenna Arrays
}

\author{
David J. Sadler, Member, IEEE, and Athanassios Manikas, Senior Member, IEEE
}

\begin{abstract}
In this paper, an asynchronous, multicarrier directsequence code-division multiple-access (DS-CDMA) array receiver is proposed based upon blind, composite channel vector estimation. Due to the fact that the combined effect of all received paths is estimated, the total number of paths can be greater than the number of antennas in the receiver array. Furthermore, the proposed approach is not limited by multipath coherency. The receiver is classified as an interference cancelling zero-forcing receiver, and multicarrier operation in a frequency-selective channel means that its performance can exceed that of a single-carrier array DS-CDMA. The effectiveness of the proposed approach, even in the presence of strong interference, is demonstrated by computer simulation studies.
\end{abstract}

Index Terms-Antenna arrays, blind receivers, diversity methods, interference cancellation, multicarrier CDMA, spacetime processing.

\section{NOMENCLATURE}

$\begin{array}{ll}a & \text { Scalar. } \\ \underline{a} & \text { Vector. } \\ \mathbb{A} & \text { Matrix. } \\ \square_{N} & N \times N \text { identity matrix. } \\ \underline{0}_{N} & N \text { element vector of zeros. } \\ (\cdot)^{T} & \text { Transpose. } \\ (\cdot)^{H} & \text { Hermitian transpose. } \\ (\cdot)^{\dagger} & \text { Pseudoinverse. } \\ \otimes & \text { Kronecker product. } \\ \exp (\underline{a}) & \text { Elementwise exponential of vector } \underline{a} . \\ \mathcal{L}\{\cdot\} & \text { Range space. } \\ \operatorname{dim}\{\cdot\} & \text { Dimension. } \\ \|\cdot\| & \text { Euclidean norm. } \\ \lfloor\cdot\rfloor & \text { Round down to integer. } \\ \mathcal{N} & \text { Set of natural numbers. } \\ \mathcal{Z} & \text { Set of integer numbers. } \\ \mathcal{C} & \text { Field of complex numbers. }\end{array}$

\section{INTRODUCTION}

$\mathbf{F}$ OR mobile radio systems, diversity reception is vital to counteract the detrimental effect of fading radio channels. Several varieties of multicarrier (MC) code-division multipleaccess (CDMA) radio systems have been proposed with the aim of enhancing diversity reception as compared to single-carrier,

Manuscript received June 19, 2002; revised September 17, 2002; accepted September 19, 2002. The editor coordinating the review of this paper and approving it for publication is A. Svensson.

The authors are with the Communications and Signal Processing Research Group, Department of Electrical and Electronic Engineering, Imperial College London, London SW7 2BT, U.K. (e-mail: a.manikas@ic.ac.uk).

Digital Object Identifier 10.1109/TWC.2003.819025 direct-sequence (DS) CDMA systems [1]. If DS-CDMA signalling is used then path diversity is accessed by coherently combining temporally spread paths that arrive at the receiver [2]. Alternatively, for multicarrier systems where each baseband symbol is assigned to multiple subcarriers for parallel transmission, there is the opportunity to realize a multicarrier diversity gain if the fading processes associated with different subcarriers are not fully correlated. This situation will arise when subcarriers are sufficiently separated in frequency. Depending on the length of the delay spread, the diversity available with singlecarrier DS-CDMA may be inadequate, particularly for a channel with a typical decaying multipath intensity profile where longer delay paths contain relatively little power. An MC-DS-CDMA system operating within the same total bandwidth as the singlecarrier system can produce better performance, even though the order of diversity of the channel is the same in both cases. This is because the diversity gain actually obtained at the receiver can be greater for multicarrier signalling. It is noted that multicarrier operation reduces the number of resolvable paths in each subchannel but more power can be recovered from each resolvable path, as is properly supported by the theoretical work presented in [3].

Much of the existing work on MC-DS-CDMA only considers narrowband subchannels. A consequence of this assumption is that each subchannel experiences flat fading, so the maximum order of diversity is limited to the total number of subcarriers. Furthermore, to achieve nonselective subchannels requires a sufficiently long chip interval relative to the delay spread of the channel. Such a restriction on the maximum chip rate places an upper limit on the symbol rate for the situation when a particular level of processing gain is required. The receiver described in [4] has a correlator for each subcarrier, and the outputs of the correlators are combined with a maximum ratio combiner. Consequently, diversity gain is realized without a RAKE receiving structure. Minimum mean square error (MMSE) detection of MC-DS-CDMA is considered in [5] which provides diversity while reducing the multiple access interference (MAI) due to the cochannel users. Furthermore, a blind MMSE receiver is proposed in [6] where subspace techniques are applied to estimate the required weight vector without the use of a training sequence.

If subchannels are permitted to be frequency-selective then there is no inherent limitation on the chip rate, and bit-error rate (BER) performance gains are possible. In [7], MC-DS-CDMA is analyzed when each subchannel is subject to frequency-selective fading. Multipath is a source of path diversity, but it also creates intersubcarrier interference in the correlation receiver which increases the BER. The results given in [7] indi- 


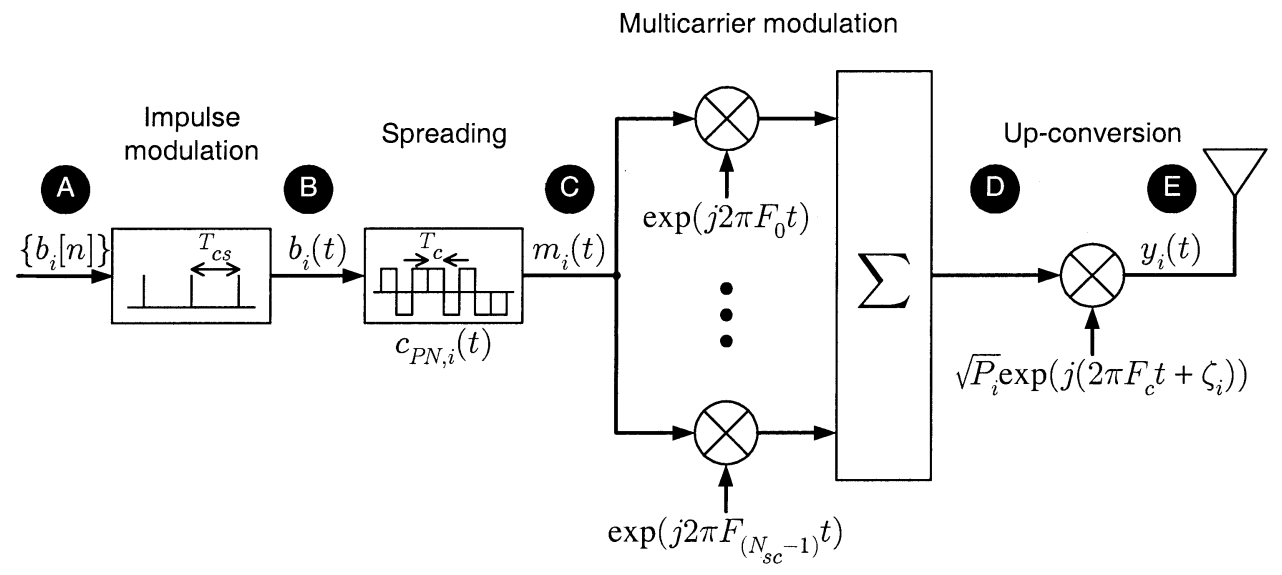

Fig. 1. Mobile transmitter block diagram.

cate that some delay spread is beneficial—the best performance is achieved when there is a balance between path and frequency diversity.

So far, single-antenna systems have been considered, however, antenna arrays can be used not only for MAI reduction, but also as a means to harness diversity from the spatial domain. Representative examples for single-carrier DS-CDMA are found in [8]-[11]. The application of arrays to multicarrier CDMA has also received some attention. In [12], MAI is partially suppressed in the spatial domain prior to detection. Code-filtering is used to estimate the beamformer weight vector, but interference is only reduced to the sidelobe level of the array pattern, rather than being completely nulled. Better results occur when processing is performed jointly in space and time, albeit with increased computational complexity. This is the case in [13] where multiuser detection significantly reduces the MAI, although channel information for all users is assumed to be available. A blind channel estimator is covered in [14] which utilizes a subspace method. However, the approach is limited by the assumption of frequency nonselective subchannels and restricts the array to be linear with a uniform element spacing.

Adaptive reception of MC-DS-CDMA with spatial diversity is explored in [15], where a vector to reduce MAI, while combining contributions from different subcarriers, is found by taking the principle eigenvector of the received signal covariance matrix. The weight vector is calculated without knowledge of the channel or timing of the cochannel users. An alternative receiver is proposed in [16] where each subcarrier has a beamforming weight applied prior to diversity combination of the subcarriers, and a performance improvement over single-carrier DS-CDMA is reported. Both of these approaches use joint space-time processing, however, it is assumed that each subchannel experiences flat fading, which limits system performance.

In this paper, asynchronous MC-DS-CDMA for fading, multipath dispersive subcarrier channels is investigated. An array receiver is employed at the base station so that processing is performed jointly in the spatial, temporal and multicarrier domains, producing a significant performance enhancement. A blind, subspace technique is proposed which uses the concept of alter- nating projection to iteratively converge upon a reliable estimate for the composite channel vector of a particular user. The projection method has previously been applied for single-antenna DS-CDMA, but only for MAI suppression in single-path channels [17]. A blind zero-forcing receiver can be calculated using the channel information, which provides diversity reception and asymptotically complete MAI cancellation. Simulations confirm that the performance of the proposed system can exceed that of single-carrier array DS-CDMA.

The rest of the paper is organized as follows: In Section II, a comprehensive model is developed which is used in Section III, where the blind, composite channel vector estimator is derived and the receiver weight vector is discussed. In Section IV, computer simulations are presented to evaluate MC-DS-CDMA and demonstrate blind receiver performance. Finally, the paper is concluded in Section V.

\section{SYSTEM MODEL}

In this section, an asynchronous MC-DS-CDMA spatio-temporal array communication system subject to a frequency-selective channel is described, focussing on the uplink. The signal received at the base station array is modeled and shown to contain significant interference as well as components of the desired signal.

\section{A. Transmitter}

Consider the block diagram of a particular mobile transmitter in Fig. 1. At point A, the $i$ th user produces a sequence of complex channel symbols according to the $\mathcal{M}$-ary modulation scheme to be employed, where $\mathcal{M}$ is the number of points in the signal constellation. The channel symbols are denoted $\left\{b_{i}[n] \in \mathcal{C}, \forall n \in \mathcal{Z}\right\}$ and have a rate of $r_{c s}=r_{b} / \log _{2}(\mathcal{M})$ symbols per second. In this paper, quaternary phase shift keying (QPSK) is considered — each symbol being imparted with unit energy.

The channel symbol sequence is transformed into an impulse train at point $\mathrm{B}$ given by

$$
b_{i}(t)=\sum_{n=-\infty}^{+\infty} b_{i}[n] \delta\left(t-n T_{c s}\right), \quad n T_{c s} \leq t<(n+1) T_{c s}
$$




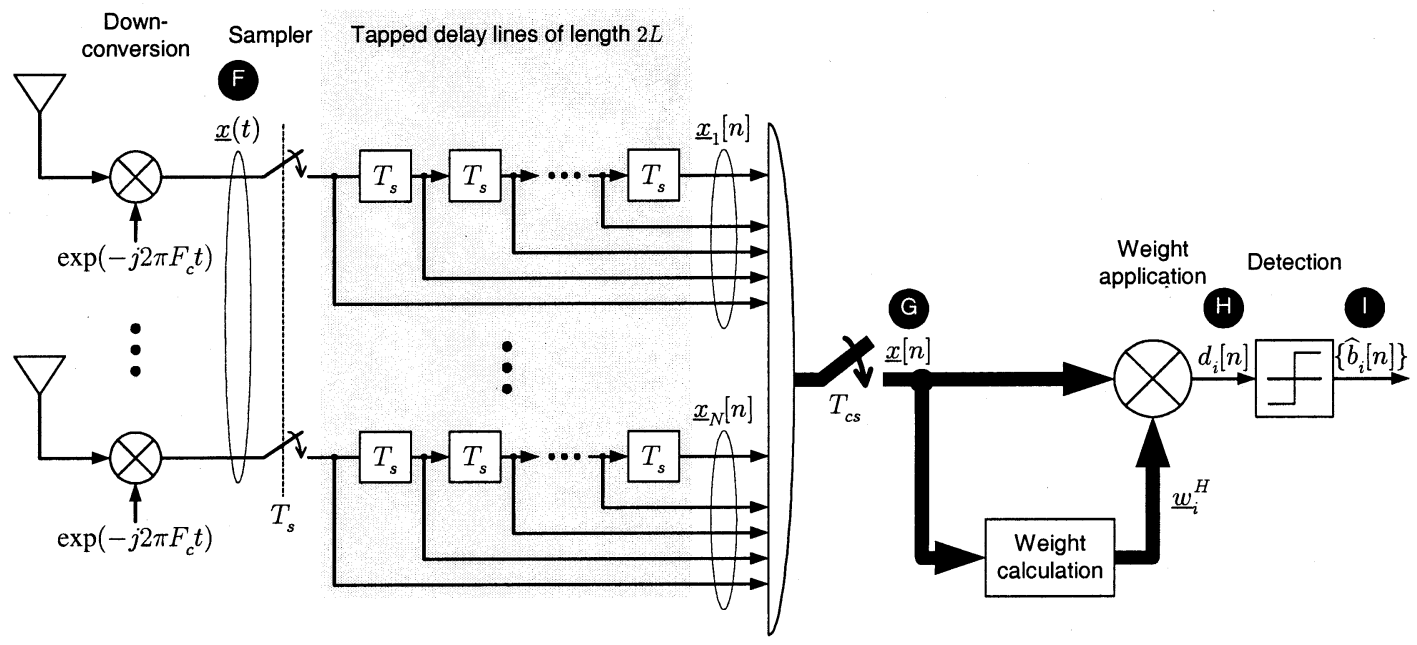

Fig. 2. Base station array receiver block diagram.

where $T_{c s}=1 / r_{c s}$ is the channel symbol period and $\delta(t)$ is the delta function. Convolving this signal with one period of a pseudo-noise (PN) signal spreads the signal over a wider bandwidth, producing a baseband DS-CDMA signal at point $\mathrm{C}$

$$
m_{i}(t)=\sum_{n=-\infty}^{+\infty} b_{i}[n] c_{\mathrm{PN}, i}\left(t-n T_{c s}\right) .
$$

In (2), a single period of the PN-signal for the $i$ th user is modeled by

$$
\begin{aligned}
c_{\mathrm{PN}, i}(t) & =\sum_{m=0}^{N_{c}-1} \alpha_{i}[m] p_{c}\left(t-m T_{c}\right), \\
m T_{c} & \leq t<(m+1) T_{c}
\end{aligned}
$$

where $\left\{\alpha_{i}[m] \in\{-1,+1\}, m=0,1, \ldots, N_{c}-1\right\}$ is the $i$ th user's PN-sequence of length $N_{c}$ chips and $p_{c}(t)$ is a rectangular, chip pulse waveform of duration $T_{c}$. Note that a short code system is being used, so the number of chips per symbol is equal to the length of the PN-sequence.

The DS-CDMA signal now modulates $N_{s c}$ subcarriers, with the baseband frequency of the $k$ th subcarrier relative to the lowest subcarrier frequency being $F_{k}=F_{0}+k \cdot \Delta F$ where $k=0,1, \ldots, N_{s c}-1$, and the subcarrier separation is set to $\Delta F=V / T_{c}, V \in \mathcal{N}$. The modulated subcarriers are summed to produce the signal at point $\mathrm{D}$ which is then upconverted to the carrier frequency to produce the transmitted radio frequency signal at point $\mathrm{E}$

$$
\begin{aligned}
y_{i}(t)=\sum_{k=0}^{N_{s c}-1} \sqrt{P_{i}} \exp \left(j\left(2 \pi F_{c} t+\zeta_{i}\right)\right) \\
\cdot \exp \left(j 2 \pi F_{k} t\right) m_{i}(t)
\end{aligned}
$$

in which $P_{i}$ is the transmitted power, $F_{c}$ is the carrier frequency, and $\zeta_{i}$ is a random phase offset relative to the base station receiver. Complex notation is used throughout this paper, with the understanding that a quadrature modulator and demodulator are used in a practical implementation.

\section{B. Channel Model}

The radio channel for each subcarrier is assumed to be fading and multipath dispersive so that the array complex baseband channel impulse response for the $k$ th subcarrier, $j$ th path of the $i$ th user can be represented by

$$
\underline{c}_{i j k}(t)=\beta_{i j k} \underline{S}_{i j k} \delta\left(t-\tau_{i j}\right) .
$$

$\beta_{i j k}$ is the complex path coefficient which encompasses a random phase shift as well as fading. The magnitude of $\beta_{i j k}$ is typically modeled using a Rayleigh probability density function (pdf) for nonline of sight propagation. $\tau_{i j}$ is the path delay and will be the same for all subcarriers, for a particular path. The vector $\underline{S}_{i j k} \triangleq \underline{S}\left(\theta_{i j}, F_{k}\right)$ is the array manifold vector at a frequency of $F_{c}+F_{k}$ for a path arriving at an azimuth angle of $\theta_{i j}$. Without a loss of generality, the directional considerations are restricted to the $x-y$ plane, so elevation has been ignored. The form of $\underline{S}_{i j k}$ for an $N$ element array of isotropes is

$$
\underline{S}_{i j k}=\exp \left(\frac{-j 2 \pi\left(F_{c}+F_{k}\right)}{c}\left[\underline{r}_{1}, \underline{r}_{2}, \ldots, \underline{r}_{N}\right]^{T} \underline{u}_{i j}\right)
$$

where the vectors $\underline{r}_{1}, \underline{r}_{2}, \ldots, \underline{r}_{N}$ contain the antenna positions in meters using Cartesian coordinates, $\underline{u}_{i j}=$ $\left[\cos \left(\theta_{i j}\right), \sin \left(\theta_{i j}\right), 0\right]^{T}$ is a unit vector pointing in the direction $\theta_{i j}$, and $c$ is the speed of light.

In general, the parameters $\beta_{i j k}, \underline{S}_{i j k}$, and $\tau_{i j}$ can be assumed to be independent of time for symbols transmitted during the channel coherence time. For this case, the channel is assumed to be quasistationary.

\section{Array Receiver Front End}

At the base station antenna array the superimposed radio signals for all users, paths and subcarriers will be received. $M$ mobile stations are considered, with the channel for the $i$ th user having a total of $K_{i}$ resolvable paths. After removal of the carrier the $N \times 1$ complex received signal vector at point $\mathrm{F}$ in Fig. 2 is

$$
\begin{aligned}
\underline{x}(t)=\sum_{i=1}^{M} \sum_{j=1}^{K_{i}} \sum_{k=0}^{N_{s c}-1} \beta_{i j k} \underline{S}_{i j k} \\
\cdot \exp \left(j 2 \pi F_{k}\left(t-\tau_{i j}\right)\right) m_{i}\left(t-\tau_{i j}\right)+\underline{n}(t)
\end{aligned}
$$

where $\beta_{i j k}$ has absorbed the complex factor $\sqrt{P_{i}} \exp$ $\left(j\left(\zeta_{i}-2 \pi F_{c} \tau_{i j}\right)\right)$, and $\underline{n}(t)$ represents complex additive white Gaussian noise (AWGN). The noise vector, assumed 
to be temporally and spatially uncorrelated, models isotropic noise.

The signal at point $\mathrm{F}$ is then discretized with a bank of samplers operating at a rate of $1 / T_{s}$ where $T_{s}=T_{c} / q N_{s c}$ and $q \in \mathcal{N}$ is the oversampling factor. This implies that the delay $\tau_{i j}$ is modeled as $\tau_{i j}=\left(l_{i j}+\rho_{i j}\right) T_{s}$ with $l_{i j} \in\{0,1, \ldots, L-1\}$ and $\rho_{i j} \in[0,1)$, where $L=q N_{c} N_{s c}$ is the number of temporal samples per symbol. This means that the model can accommodate delays, $\tau_{i j}$, that lie within the range $\left[0, T_{c s}\right)$. The restriction is made to reduce the complexity of the rest of the modeling process but is not an inherent limitation of the system.

The samples pass into a bank of $N$ tapped delay lines of length $2 L$. A long vector $\underline{x}[n]$ is formed at point $\mathrm{G}$ by concatenating the contents of the tapped delay lines for all antennas and reading the entries every symbol period

$$
\begin{aligned}
& \underline{x}[n]=\left[\underline{x}_{1}[n]^{T}, \underline{x}_{2}[n]^{T}, \ldots, \underline{x}_{N}[n]^{T}\right]^{T} \in \mathcal{C}^{2 N L \times 1} \\
& \underline{x}[n]=\sum_{i=1}^{M} \sum_{j=1}^{K_{i}} \sum_{K=0}^{N_{s c}-1} \beta_{i j k} \underline{S}_{i j k} \\
& \otimes(\underbrace{\left(\mathbb{J}^{T}\right)^{L} \boldsymbol{J}^{l_{i j}} \underline{\mathfrak{a}}_{i k}\left[l_{i j}\right] b_{i}[n-1]}_{\text {previous }} \\
& +\underbrace{\mathfrak{l}^{l_{i j}} \underline{\mathfrak{a}}_{i k}\left[l_{i j}\right] b_{i}[n]}_{\text {current }} \\
& +\underbrace{\jmath^{L} \underline{\jmath}^{l_{i j}} \underline{\mathfrak{a}}_{i k}\left[l_{i j}\right] b_{i}[n+1]}_{\text {next }}) \\
& +\underline{n}[n] \text {. }
\end{aligned}
$$

This multicarrier space-time received signal vector contains the signals associated with the $n$th (current) symbol, furthermore, contributions from the previous and next data symbols are present due to the lack of synchronization. A complete representation is given by (9), in which $\otimes$ is the Kronecker product and $\underline{n}[n]$ is discrete complex AWGN. The path coefficient, $\beta_{i j k}$, has additionally absorbed $\exp \left(-j 2 \pi F_{k} \rho_{i j} T_{s}\right)$ which is the complex factor due to the fractional path delay, $\rho_{i j} T_{s}$. Furthermore

$$
\underline{\mathfrak{a}}_{i k}[l]=\left[\mathfrak{a}_{i k}[0, l], \mathfrak{a}_{i k}[1, l], \ldots, \mathfrak{a}_{i k}[L-1, l], \underline{0}_{L}^{T}\right]^{T}
$$

is the temporal vector for the $k$ th subcarrier of the $i$ th user, for paths arriving with an integer delay of $l$ sample periods, with elements defined by

$$
\mathfrak{a}_{i k}[m, l]=\alpha_{i}\left[\left\lfloor\frac{m}{q N_{s c}}\right\rfloor\right] \exp \left(j 2 \pi F_{k}(m-l) T_{s}\right)
$$

which contains the PN-code $\alpha_{i}$. In (9), $\mathbb{I}$ is a $(2 L \times 2 L)$ shift matrix which is used to represent the different multipath delays in a compact way. It is defined as

$$
J=\left[\begin{array}{ccccc}
0 & 0 & \cdots & 0 & 0 \\
1 & 0 & \cdots & 0 & 0 \\
0 & 1 & \cdots & 0 & 0 \\
\vdots & \vdots & \ddots & \vdots & \vdots \\
0 & 0 & \cdots & 1 & 0
\end{array}\right]=\left[\begin{array}{cc}
\underline{0}_{2 L-1}^{T} & 0 \\
\mathbb{\rrbracket}_{2 L-1} & \underline{0}_{2 L-1}
\end{array}\right] .
$$

For example, if $\mathbb{l}^{l}$ is applied to a column vector then it shifts the elements of the vector down by $l$ elements. Likewise, $\left(\rrbracket^{T}\right)^{l}$ up-shifts the vector by $l$ elements.

The multicarrier spatio-temporal array (STAR) manifold vector for the $k$ th subcarrier, $j$ th path of the $i$ th user is now defined

$$
\underline{\mathfrak{h}}_{i j k} \triangleq \underline{S}_{i j k} \otimes\left(\mathfrak{J}^{l_{i j}} \underline{\mathfrak{a}}_{i k}\left[l_{i j}\right]\right) \in \mathcal{C}^{2 N L \times 1} .
$$

This STAR manifold vector can be seen to depend entirely upon the $i$ th user's PN-code and the direction and delay of the $j$ th path for a particular subcarrier. The user code is already known at the receiver, conversely, the direction and delay parameters have to be estimated if they are required. Using this definition, $\underline{x}[n]$ can be written more compactly as

$$
\begin{aligned}
& \underline{x}[n]=\sum_{i=1}^{M}\left[\mathbb{H}_{i}^{(\text {prev })} \underline{\beta}_{i}, \mathbb{H}_{i} \underline{\beta}_{i}, \mathbb{H}_{i}^{(\text {next })} \underline{\beta}_{i}\right]\left[\begin{array}{c}
b_{i}[n-1] \\
b_{i}[n] \\
b_{i}[n+1]
\end{array}\right] \\
& +\underline{n}[n]
\end{aligned}
$$

where the matrix $\mathbb{H}_{i} \in \mathcal{C}^{2 N L \times K_{i} N_{s c}}$ has columns containing the multicarrier STAR manifold vectors $\underline{\mathfrak{h}}_{i j k} \forall j, k$. Furthermore

$$
\begin{aligned}
& \mathfrak{H}_{i}^{(\text {prev })}=\left(\rrbracket_{N} \otimes\left(\mathbb{J}^{T}\right)^{L}\right) \mathbb{H}_{i} \\
& \mathbb{H}_{i}^{(\text {next })}=\left(\rrbracket_{N} \otimes \mathbb{J}^{L}\right) \mathbb{H}_{i}
\end{aligned}
$$

and the vector $\beta, \in \mathcal{C}^{K_{i} N_{s c} \times 1}$ in (14), contains the path coefficients $\beta_{i j k} \forall j, k$.

Taking user-1 as the desired user to be detected, the data vector can be split up into four factors

$$
\underline{x}[n]=\mathbb{H}_{1} \underline{\beta}_{1} b_{1}[n]+\underline{\eta}_{\mathrm{ISI}}[n]+\underline{\eta}_{\mathrm{MAI}}[n]+\underline{n}[n]
$$

where $\mathbb{H}_{1} \underline{\beta}_{1} b_{1}[n]$ is the wanted signal component and the intersymbol interference (ISI) and MAI are given by

$$
\begin{aligned}
\underline{\eta}_{\mathrm{ISI}}[n]= & {\left[\mathbb{H}_{1}^{(\text {prev })} \underline{\beta}_{1}, \mathbb{H}_{1}^{(\text {next })} \underline{\beta}_{1}\right]\left[\begin{array}{l}
b_{1}[n-1] \\
b_{1}[n+1]
\end{array}\right] } \\
\underline{\eta}_{\mathrm{MAI}}[n]= & \sum_{i=2}^{M}\left[\mathbb{H}_{i}^{(\text {prev })} \underline{\beta}_{i}, \mathbb{H}_{i} \underline{\beta}_{i}, \mathbb{H}_{i}^{(\text {next })} \underline{\beta}_{i}\right] \\
& \times\left[\begin{array}{c}
b_{i}[n-1] \\
b_{i}[n] \\
b_{i}[n+1]
\end{array}\right] .
\end{aligned}
$$

\section{Linear Receiver Structure}

At the input of the decision device (point $\mathrm{H}$ ) in Fig. 2 a decision variable is observed by the application of the weight vector $\underline{w}_{1} \in \mathcal{C}^{2 N L \times 1}$ associated with user-1. This vector should be designed to suppress interference-whether it is MAI or ISI-while despreading and coherently combining energy from different paths and subcarriers. Additionally, the output noise power will be maintained at the same level as the input noise power. The decision variable for the $n$th symbol of user- 1 is

$$
d_{1}[n]=\underline{w}_{1}^{H} \underline{x}[n] .
$$

\section{THEORETICAL FRAMEWORK}

The structure of the system model is now exploited for estimation of channel information from received blocks of data, without recourse to the use of training signals. Consequently, efficiency is maximized because a portion of the signalling 
time does not need to be allocated to training. The composite channel vector for the current symbol of the $i$ th user is defined as $\underline{h}_{i} \triangleq \mathbb{H}_{i} \underline{\beta}_{i}$ and contains all of the effects of the radio channel applied to symbol $b_{i}[n]$. The subspaces that contain this channel vector are important to our estimation approach. Specifically, two nonorthogonal subspaces will be identified under the constraint that the one-dimensional (1-D) subspace spanned by $\underline{h}_{i}$ is the intersection of these two subspaces.

\section{A. Subspace Constraints}

One of the two constraint subspaces can be identified from the second-order statistics of $\underline{x}[n]$ described in (14). By eigendecomposition of the covariance matrix, $\mathbb{R}_{x x}$, the matrix $\mathbb{E}_{s}$ is formed, which has the $3 M$ normalized signal eigenvectors as its columns, and is a basis for the signal subspace. In (14), the term related to the current symbol for the $i$ th user is $\mathbb{H}_{i} \underline{\beta}_{i} b_{i}[n]$, where it is clear that the columns of $\mathfrak{H}_{i}$ are linearly combined by the vector of path coefficients $\beta_{i}$, so that the contribution of $b_{i}[n]$ to the signal subspace is only comprised of a single dimension. Therefore, the range space of $\mathbb{E}_{s}$ contains all of the 1-D subspaces spanned by the composite channel vectors of all users, thus providing the following signal subspace constraint:

$$
\mathcal{L}\left\{\underline{h}_{i}\right\}=\mathcal{L}\left\{\mathbb{H}_{i} \underline{\beta}_{i}\right\} \in \mathcal{L}\left\{\mathbb{E}_{s}\right\}
$$

The matrix projector into the signal subspace is given by

$$
\mathbb{P}_{s}=\mathbb{E}_{s} \mathbb{E}_{s}^{H}
$$

since the columns of $\mathbb{E}_{s}$ are orthonormal.

In order to identify the second subspace constraint based on the code for the $i$ th user and the structure of the multicarrier signal, the code submatrix of the $k$ th subcarrier for paths arriving with an integer delay of $\ell T_{s}$ is defined as

$$
\mathbb{C}_{i k}[\ell]=\rrbracket_{N} \otimes\left(\mathbb{I}^{\ell} \underline{\mathfrak{a}}_{i k}[\ell]\right) \in \mathcal{C}^{2 N L \times N} .
$$

The overall code matrix for the $i$ th user is denoted $\mathbb{C}_{i} \in \mathcal{C}^{2 N L \times N N_{s c} L}$ and is created by concatenating all of the submatrices $\mathbb{C}_{i k}[\ell]$ for $k=0,1, \ldots,\left(N_{s c}-1\right)$ and $\ell=0,1, \ldots,(L-1)$. Additionally, the composite array response subvector of the $k$ th subcarrier is

$$
\underline{\mathfrak{s}}_{i k}[\ell]=\sum_{\text {all paths with } l_{i j}=\ell} \beta_{i j k} \underline{S}_{i j k} .
$$

It should be noted that for any particular integer delay $\ell T_{s}$ there may be no paths present, a single path, or multiple paths. The total composite array response vector for the $i$ th user, $\underline{\mathfrak{s}}_{i} \in$ $\mathcal{C}^{N N_{s c} L \times 1}$, is formed by stacking up all of the subvectors $\underline{\mathfrak{s}}_{i k}[\ell]$ for $k=0,1, \ldots,\left(N_{s c}-1\right)$ and $\ell=0,1, \ldots,(L-1)$.

Using the above definitions it is possible to represent the composite channel vector as

$$
\underline{h}_{i}=\mathbb{H}_{i} \underline{\beta}_{i}=\mathbb{C}_{i} \underline{\mathfrak{s}}_{i} .
$$

In (23), it is apparent that $\underline{h}_{i}$ lies in the range space of $\mathbb{C}_{i}$ so the columns of the matrix $\mathbb{C}_{i}$ define the following code subspace constraint:

$$
\mathcal{L}\left\{\underline{h}_{i}\right\}=\mathcal{L}\left\{\mathbb{C}_{i} \underline{\mathfrak{s}}_{i}\right\} \in \mathcal{L}\left\{\mathbb{C}_{i}\right\}
$$

The operator that projects into this subspace is generated by

$$
\mathbb{P}_{i}=\mathbb{C}_{i}\left(\mathbb{C}_{i}^{H} \mathbb{C}_{i}\right)^{\dagger} \mathbb{C}_{i}^{H}
$$

where the pseudoinverse operation is required because $\mathbb{C}_{i}$ does not have full column rank. ${ }^{1}$

\section{B. Estimation Algorithm and Convergence Issues}

The approach proposed to estimate the composite channel vector applies the theorem of alternating projection to find the intersection of the two constraint subspaces [18]. Alternating projection is an iterative technique with strong convergence properties that can generally be applied when any two subspaces in a Hilbert space intersect. The theorem is explained in a more general context in [19]. The estimate $\widehat{\underline{h}}_{i}$ will approximate $\underline{h}_{i}$ up to a complex scaling factor, i.e., $\underline{\underline{h}}_{i} \approx \gamma_{i} \underline{h}_{i}$ with $\gamma_{i} \in \mathcal{C}$. Formulating the alternating projection algorithm

$$
\underline{\hat{h}}_{i}^{[p+1]}=\mathbb{P}_{i} \mathbb{P}_{s} \underline{\hat{h}}_{i}^{[p]}
$$

with the initial guess $\underline{\hat{h}}_{i}^{[0]}$ arbitrarily chosen but nonzero.

Convergence of the alternating projection algorithm is guaranteed. However, convergence alone does not ensure that the estimate of $\underline{h}_{i}$ will indeed be a scaled version of the true vector. As previously mentioned, the condition for convergence to a unique solution is

$$
\mathcal{L}\left\{\underline{h}_{i}\right\}=\mathcal{L}\left\{\mathbb{C}_{i}\right\} \cap \mathcal{L}\left\{\mathbb{E}_{s}\right\}
$$

which means that the two constraint subspaces only intersect at a line in the overall $2 N L$-dimensional observation space, i.e., $\operatorname{dim}\left\{\mathcal{L}\left\{\mathbb{C}_{i}\right\} \cap \mathcal{L}\left\{\mathbb{E}_{s}\right\}\right\}=1$. A necessary condition for (27) to hold is that the total number of linear equations equals or exceeds the number of unknowns minus one. The dimension of the left nullspace of the code constraint matrix indicates that it provides $N L-N\left(N_{s c}-1\right)$ independent equations. Likewise, the subspace constraint provides a total of $2 N L-3 M$ independent equations. This implies that the following inequality must be true

$$
3 M \leq N\left(L-N_{s c}+1\right)+1
$$

which can be ensured by increasing the number of antennas or subcarriers. Extensive simulation has shown that (27) does generally hold if (28) is obeyed, even for fully loaded systems.

An alternative procedure can be applied to avoid iterative estimation of the composite channel vector. As $p \rightarrow \infty$ then $\underline{\hat{h}}_{i}^{[p]} \rightarrow \underline{\hat{h}}_{i}^{[\infty]}$, and (26) can be written as

$$
\underline{\hat{h}}_{i}^{[\infty]}=\mathbb{P}_{i} \mathbb{P}_{s} \underline{\hat{h}}_{i}^{[\infty]} .
$$

The final estimate, $\underline{\hat{h}}_{i}^{[\infty]}=\gamma_{i} \underline{h}_{i}$, is equal to the eigenvector of matrix $\mathbb{P}_{i} \mathbb{P}_{s}$ which has a corresponding eigenvalue of one. This direct method provides the required solution in a single step, but it may be more computationally complex than the iterative method in a practical implementation.

\section{Weight Vector Construction}

If user- 1 is considered to be the desired user then the blind estimate of $\underline{h}_{1}$ will facilitate the following multicarrier spatiotemporal (3D) RAKE receiver

$$
\underline{w}_{1}=\kappa_{1} \underline{\hat{h}}_{1}
$$

\footnotetext{
${ }^{1}$ The pseudoinverse here is defined based on the singular value decomposi-
} 
where $\kappa_{1}$ is a normalization factor. This weight vector performs maximum ratio combining by coherently combining all paths, for all subcarriers, of user-1. The 3D RAKE receiver is an extension of the standard spatio-temporal (2D) RAKE [8] but with an additional multicarrier dimension. It is also equivalent to the MC-RAKE of [3] extended to include spatial processing.

Such a weight vector maximizes the SNR criterion and is optimum when there is no interference. However, in a situation where there is significant interference, the performance of the 3D RAKE receiver is not satisfactory.

Additional estimation of all users composite channel vectors, $\widehat{\underline{h}}_{i} \forall i$, means that a more advanced blind receiver can be formulated which reduces MAI and ISI

$$
\underline{w}_{1}=\kappa_{1} \mathbb{P}_{\widehat{\mathbb{H}}_{\text {int }}}^{\perp} \widehat{\underline{h}}_{1} \text {. }
$$

The complement projection matrix $\mathbb{P}_{\mathbb{\mathbb { H }}_{\text {int }}}^{\perp}$ projects the estimated composite channel vector for user- 1 to be orthogonal to the subspace occupied by the MAI and ISI, i.e., the orthogonal complement of the subspace spanned by the columns of matrix $\widehat{\mathbb{H}}_{\text {int }}$. The estimated interference matrix is given by

$$
\widehat{\mathbb{H}}_{\text {int }}=\left[\left(\mathbb{\rrbracket}_{N} \otimes\left(J^{T}\right)^{L}\right) \widehat{\mathbb{H}}, \overline{\mathbb{H}}_{1},\left(\mathbb{\rrbracket}_{N} \otimes J^{L}\right) \hat{\mathbb{H}}\right]
$$

where

$$
\begin{aligned}
\widehat{\mathbb{H}} & =\left[\widehat{\widehat{h}}_{1}, \hat{\widehat{h}}_{2}, \ldots, \widehat{\widehat{h}}_{M}\right] \in \mathcal{C}^{2 N L \times M} \\
\overline{\mathbb{H}}_{1} & =\left[\underline{\widehat{h}}_{2}, \widehat{\underline{h}}_{3}, \ldots, \underline{\underline{h}}_{M}\right] \in \mathcal{C}^{2 N L \times(M-1)} .
\end{aligned}
$$

To generate the complement projection matrix the following expression is used:

$$
\mathbb{P}_{\widehat{\mathbb{H}}_{\text {int }}}^{\perp}=\mathbb{I}_{2 N L}-\widehat{\mathbb{H}}_{\text {int }}\left(\widehat{\mathbb{H}}_{\text {int }}^{H} \widehat{\mathbb{H}}_{\text {int }}\right)^{-1} \widehat{\mathbb{H}}_{\text {int }}^{H} \text {. }
$$

The receiver described by (31) is a multicarrier STAR zero-forcing receiver which maximizes the SIR criterion and provides (asymptotically) complete interference cancellation. Alternatively, the signal-to-noise-plus-interference ratio (SNIR) criterion can be maximized by using a MMSE receiver [20]. This receiver has slightly improved performance at low SNR but additionally requires estimation of the additive noise power. $^{2}$

The complete procedure for composite channel vector estimation and construction of the zero-forcing multicarrier space-time weight vector for user-1, is summarized in Table I and depicted graphically in Fig. 3.

\section{SimUlation STUdiES}

In order to evaluate the performance of array MC-DS-CDMA and the proposed blind zero-forcing receiver, a number of computer simulations were executed. For all of the results presented in this section, Gold codes have been used for the PN-sequences. If an array is specified then it is a linear array with half wavelength antenna spacing. Differential QPSK modulation is utilized throughout, which suffers an approximate performance disadvantage of $2.3 \mathrm{~dB}$ at large $\mathrm{SNR}_{\text {in }}$ when compared to coherent QPSK, but means that absolute carrier phase information is not required. No oversampling has been used, so the parameter $q$ was set equal to one.

\footnotetext{
${ }^{2}$ In a practical situation, the noise power can be estimated as the mean of the eigenvalues associated with the noise eigenvectors.
}

TABLE I

SUMMARY OF THE BLIND RECEPTION ALGORITHM

1. $\quad$ Form the matrix $\mathbb{C}_{i}$ based on each active user's PNsequence and the multicarrier space-time structure of the system and calculate $\mathbb{P}_{i} \forall i$. Note that these projection matrices can be pre-computed and stored in memory.

2. Estimate the covariance matrix of the multicarrier space-time received vector $\underline{x}[n]$ over a frame of data containing $U$ symbols,

$$
\widehat{\mathbb{R}}_{x x}=\frac{1}{U} \sum_{n=1}^{U} \underline{x}[n] \underline{x}[n]^{H} .
$$

3. Decompose $\widehat{\mathbb{R}}_{x x}$ by eigenanalysis to estimate $\mathbb{E}_{s}$ of dimensionality $3 M$ and construct the projector into the signal subspace, $\mathbb{P}_{s}$.

4. Initialize $\underline{\widehat{\underline{h}}}_{i}^{[0]}$.

5. Calculate $\underline{\widehat{h}}_{i}^{[p+1]}=\mathbb{P}_{i} \mathbb{P}_{s} \hat{\widehat{h}}_{i}^{[p]}$.

6. Repeat step 5 for a fixed number of iterations, or until sufficient convergence has been obtained. For a practical estimator, a criterion to judge the proximity of convergence is to calculate the norm of the difference between two successive estimates. Consequently, the algorithm is terminated when the following condition holds,

$$
\left\|\widehat{\underline{h}}_{i}^{[p+1]}-\underline{\widehat{h}}_{i}^{[p]}\right\| \leq \epsilon
$$

where $\epsilon$ is a predetermined threshold that controls the accuracy of the final channel estimate.

7. Repeat steps 4 to 6 until the composite channel vectors for all users have been estimated.

8. $\quad$ Generate $\underline{w}_{1}$ according to Equation 31 .

To compare fairly the performance of systems with different numbers of subcarriers the total signalling bandwidths must be equal. For all simulations the $N_{c} N_{s c}$ product is kept approximately equal to 60 . This means that for the single-subcarrier case, Gold codes containing 63 chips are used; for two subcarrier systems, 31 chip Gold codes are used; and for four subcarrier systems, 15 chip Gold codes are used. SNIR out $_{\text {is evaluated }}$ at point $\mathrm{H}$ in Fig. 2 and can be expressed as

$$
\mathrm{SNIR}_{\text {out }}=\frac{\underline{w}_{1}^{H} \mathbb{R}_{11} \underline{w}_{1}}{\underline{w}_{1}^{H} \mathbb{R}_{n+i} \underline{w}_{1}}
$$

where $\mathbb{R}_{11}$ is the covariance matrix of user- $1, \mathbb{R}_{n+i}$ is the covariance matrix of the noise plus interference, and $\mathbb{R}_{x x}=\mathbb{R}_{11}+$ $\mathbb{R}_{n+i}$.

Initially, we consider the case when there is perfect channel information available, which will provide an upper performance limit for the proposed blind receiver. Fig. 4 contains graphs of $\mathrm{SNIR}_{\text {out }}$ plotted against $\mathrm{SNR}_{\text {in }}$ for various $3 \mathrm{D}$ RAKE and zero-forcing type receivers when there are 15 users, each subjected to a 3-path, dispersive channel. It is noted that in general additional resolvable paths will be present when the number of subcarriers are reduced. However, as supported by [3], when there is a decaying multipath intensity profile only the initial strongest paths provide significant received power, so the longer 


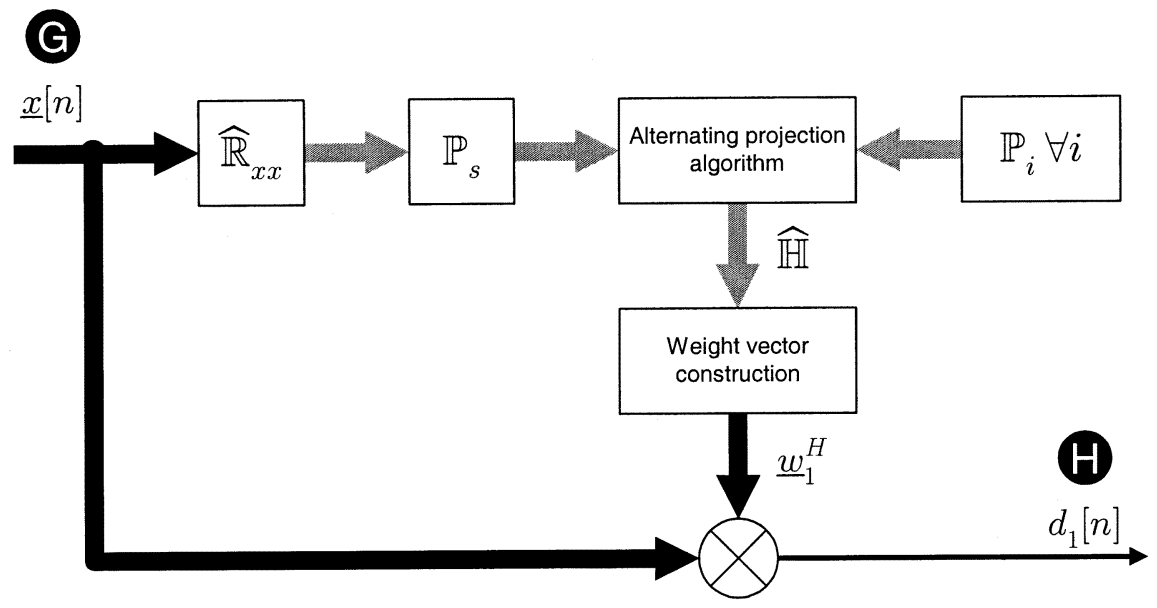

Fig. 3. Structure of the proposed blind receiver for user-1.

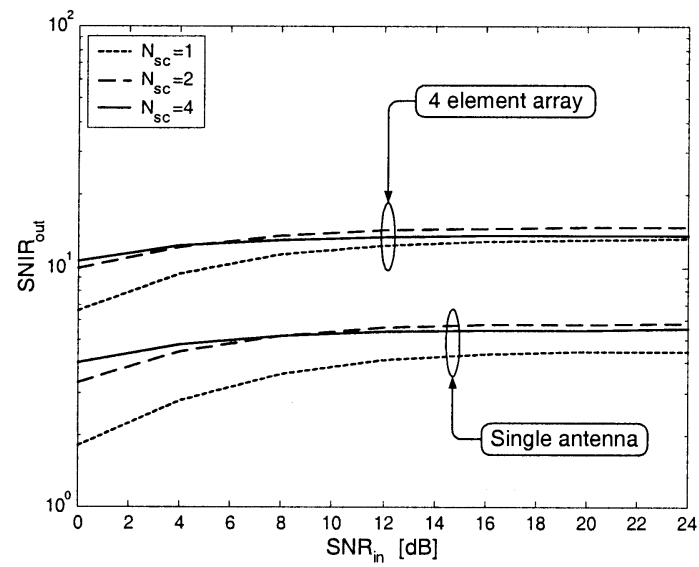

(a)

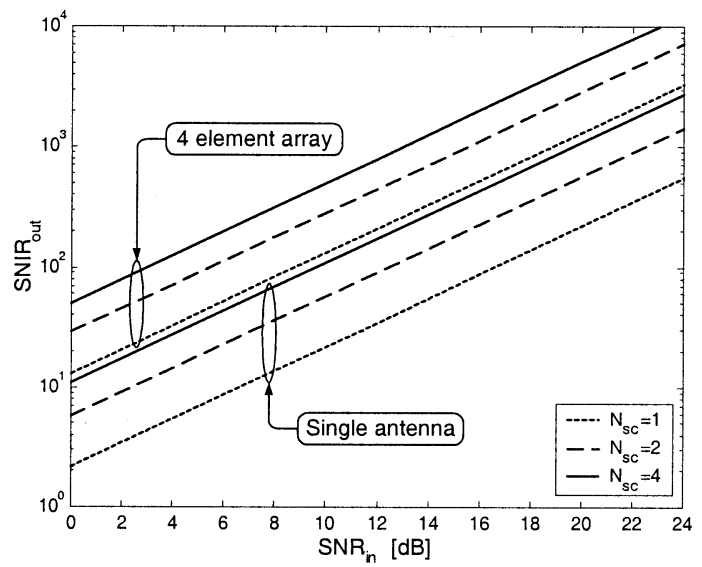

(b)

Fig. 4. Upper limit for performance of multicarrier STAR receivers when there are 15 users and perfect channel information is available, (a) 3D RAKE receiver and (b) proposed receiver.

delay but lower power (and less significant) paths have been ignored in these simulations. All paths were incident upon the array within a $120^{\circ}$ sector, with the paths for each user clustered around a nominal user direction generated from a uniform pdf within this sector. The directions of the three paths for a particular user were then generated from a Gaussian pdf with a $10^{\circ}$ standard deviation and a mean equal to the nominal user direction. Path delays were set by a uniform pdf in the range of 0 to $T_{c s}$ s. The simulation was executed for 100 bursts of 1000 bits, with the fading coefficients changed for every burst. The path coefficients for different subcarriers were evaluated independently using a Rayleigh pdf, which is the ideal case in terms of diversity. This implies that the subcarrier separation is large relative to the channel coherence bandwidth, which can be ensured during system design. Note that partial correlation of the fading in the subchannels will produce some level of performance degradation, a situation which has been studied in [3].

The results for the 3D RAKE receiver demonstrate that performance improves as the number of antennas in the base station array are increased, but the situation with regards to the number of subcarriers is less clear. It seems that the positive affect of multicarrier operation is negated by the increased interference.

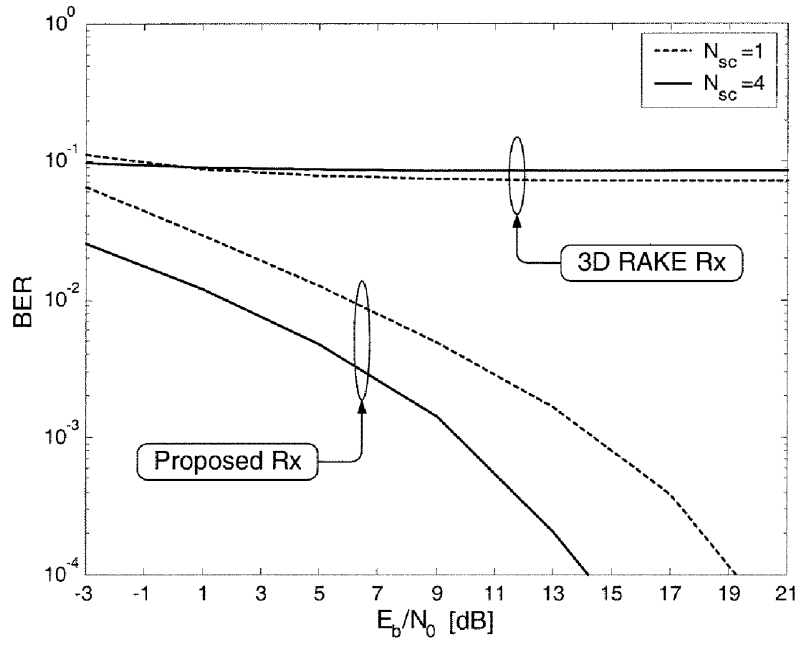

Fig. 5. BER upper limits for a four antenna system with 15 users when perfect channel information is available.

When the proposed receiver is used to cancel the interference, it becomes obvious that as the number of subcarriers increases then so does the SNIR out. Clearly, MC-DS-CDMA can outper- 


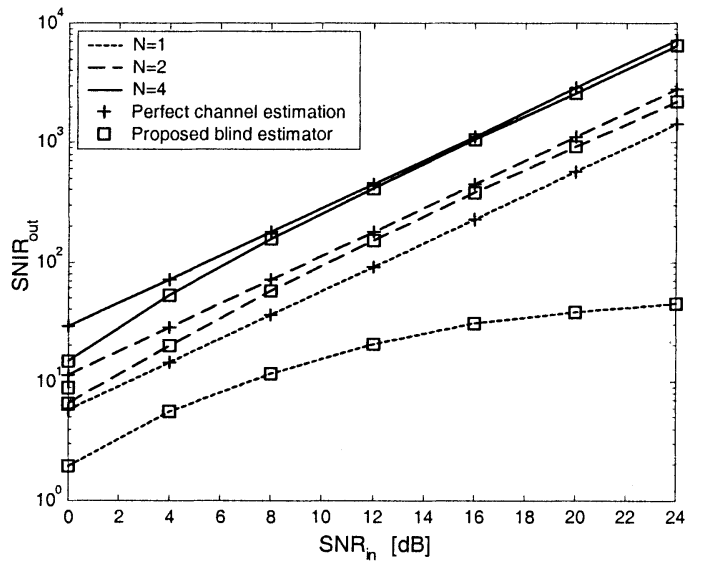

(a)

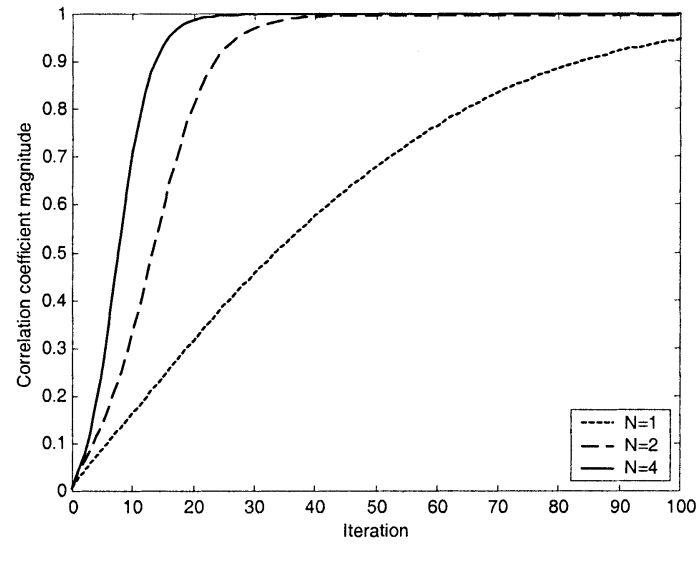

(b)

Fig. 6. Comparison of receiver performance when there are 15 users and two subcarriers are used. (a) $\mathrm{SNIR}_{\text {out }}$ and (b) channel estimation accuracy after 100 iterations.

form single carrier DS-CDMA, but the performance advantage is significant only if an interference cancelling receiver is used.

For a subset of the perfect channel information data, when a four element array is available, the BER versus energy per bit to noise spectral density ratio $\left(E_{b} / N_{0}\right)$ is plotted in Fig. 5 . The 3D RAKE receiver has an error floor which cannot be improved upon no matter how large the $E_{b} / N_{0}$. This is because the SNIR $_{\text {out }}$ saturates due to uncancelled interference. In contrast, the proposed receiver performs far better, demonstrating that interference is being removed, as well as the fading being countered by diversity combining.

When composite channel vector estimation is performed-to provide a completely blind solution to the reception problem-the proposed receiver $\mathrm{SNIR}_{\text {out }}$ against $\mathrm{SNR}_{\text {in }}$ results, for the case of two subcarriers, 31 chip codes, and 15 users, are typified by those in Fig. 6(a). The alternating projection algorithm was exited after the norm of the change between consecutive estimates dropped below $-80 \mathrm{~dB}$, or if a maximum of 100 iterations had been reached.

When there are two or four antennas in the base station array, the performance of the completely blind receiver is very close to the situation when perfect channel information is assumed. However, for the single antenna case, the blind receiver is underperforming the ideal result by a significant margin. The reason for this is explained with the help of Fig. 6(b) where the magnitude of the correlation coefficient between the estimated and actual composite channel vector is plotted for the desired user during the estimation procedure for one of the data bursts, with all of the users having the same average power and a $\mathrm{SNR}_{\text {in }}$ of $12 \mathrm{~dB}$. It can be seen that when there are two or four antennas, very high correlation is achieved within 40 or 25 iterations, respectively. In contrast, when there is only a single antenna, the convergence is much slower, and after the maximum 100 iterations the correlation between the estimated and true composite channel vectors is not sufficiently close to unity. This means that the projection operation in the proposed receiver will be imperfect, therefore, some of the MAI and ISI is still present in the decision variable, and the SNIR out will suffer accordingly. Permitting more iterations of the alternating projection algorithm

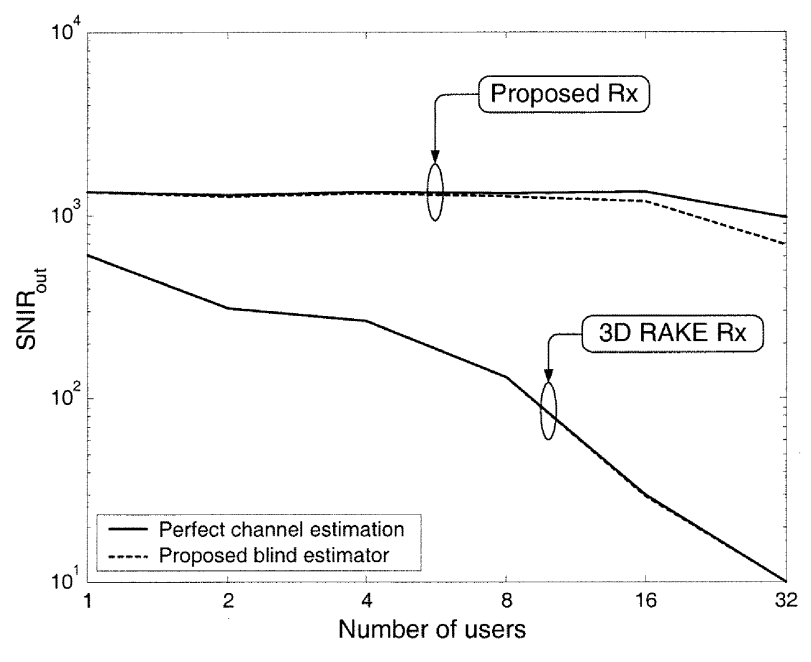

Fig. 7. System performance under a varying load factor.

will improve the channel estimate, but increasing the degrees of freedom so that convergence is accelerated (e.g., by using more antennas), is a better solution. Alternatively, the single step solution, by calculating an eigenvector of $\mathbb{P}_{i} \mathbb{P}_{s}$, could be used.

The final set of results presented in Fig. 7 demonstrate the performance of the proposed receiver as a function of the number of users. The system parameters were: $N=4, N_{s c}=2$, and $N_{c}=31$, simulated at a $\mathrm{SNR}_{\text {in }}$ of $16 \mathrm{~dB}$. As the interference increases, the SNIR out for the 3D RAKE receiver falls, but the proposed receiver has approximately constant $\mathrm{SNIR}_{\text {out }}$ regardless of the number of users. When the blind channel vector estimator is used the performance is almost the same as for the ideal case when perfect channel information is available, which demonstrates the estimator's ability to operate successfully even when there is significant interference. Applying (28) for this particular system indicates that a maximum of 81 active users may be present if the blind estimator is to be used. If this limit is exceeded then there are insufficient constraints to uniquely identify the 1-D subspace occupied by each user's composite channel vector, and in general the estimated vectors will not be equal to scaled versions of the true vectors. 


\section{CONCLUSION}

In this paper, a MC-DS-CDMA communications system for mobile use has been carefully modeled, concentrating upon the uplink channel. The base station is equipped with an array of antennas so that spatial processing, together with temporal and multi-frequency processing, can be applied. Unlike most previous work existing in this area, a fully fading multipath channel for each subcarrier is considered, as opposed to a flat fading channel.

To facilitate completely blind reception, the alternating projection algorithm is applied in a new subspace based composite channel vector estimator. This estimator uses two constraint subspaces, namely the user code subspace and the received signal subspace, to identify the 1-D space occupied by the composite channel vector of the user of interest. The estimator is resistant to interference, so will converge to a reliable result even in situations when a conventional training signal based estimator would be overwhelmed by the MAI. Additionally, the estimator is not limited to identifying a maximum number of paths, because it estimates the composite channel, which is the superposition of all incident paths for a particular user. A further advantage is that the procedure does not require the array to be well calibrated, as the array manifold is not explicitly used in the processing. Finally, a blind zero-forcing type receiver is presented and simulation results indicate that the performance obtained is very close to that of a nonblind receiver with perfect channel information.

\section{ACKNOWLEDGMENT}

The authors would like to thank Roke Manor Research Ltd. and the EPSRC for their support of this paper.

\section{REFERENCES}

[1] R. Prasad and S. Hara, "An overview of multi-carrier CDMA," in Proc. ISSSTA'96, vol. 1, 1996, pp. 107-114.

[2] J. G. Proakis, Digital Communications, 3rd ed. New York: McGrawHill, 1995.

[3] W. Xu and L. B. Milstein, "On the performance of multicarrier RAKE systems," IEEE Trans. Commun., vol. 49, pp. 1812-1823, Oct. 2001.

[4] S. Kondo and L. B. Milstein, "Performance of multicarrier DS CDMA systems," IEEE Trans. Commun., vol. 44, pp. 238-246, Feb. 1996.

[5] S. L. Miller and B. J. Rainbolt, "MMSE detection of multicarrier CDMA," IEEE J. Select. Areas Commun., vol. 18, pp. 2356-2362, Nov. 2000.

[6] J. Namgoong, T. F. Wong, and J. S. Lehnert, "Subspace multiuser detection for multicarrier DS-CDMA," IEEE Trans. Commun., vol. 48, pp. 1897-1908, Nov. 2000.

[7] E. A. Sourour and M. Nakagawa, "Performance of orthogonal multicarrier CDMA in a multipath fading channel," IEEE Trans. Commun., vol. 44, pp. 356-367, Mar. 1996.

[8] B. H. Khalaj, A. Paulraj, and T. Kailath, "2D RAKE receivers for CDMA cellular systems," in Proc. GLOBECOM'94, vol. 1, 1994, pp. 400-404.

[9] Z. Zvonar, "Combined multiuser detection and diversity reception for wireless CDMA systems," IEEE Trans. Veh. Technol., vol. 45, pp. 205-211, Feb. 1996.

[10] X. Wang and H. V. Poor, "Space-time multiuser detection in multipath CDMA channels," IEEE Trans. Signal Processing, vol. 47, pp. 2356-2374, Sept. 1999.
[11] C. B. Papadias and H. Huang, "Linear space-time multiuser detection for multipath CDMA channels," IEEE J. Select. Areas Commun., vol. 19, pp. 254-265, Feb. 2001.

[12] C. K. Kim and Y. S. Cho, "Performance of a wireless MC-CDMA system with an antenna array in a fading channel: Reverse link," IEEE Trans. Commun., vol. 48, pp. 1257-1261, Aug. 2000.

[13] P. Jung, K. Kammerlander, F. Berens, and J. Plechinger, "On multicarrier CDMA mobile radio systems with joint detection and coherent receiver antenna diversity," in Proc. UPC'96, vol. 1, 1996, pp. 61-65.

[14] X. Wu, A. Feng, and Q. Yin, "Blind space-frequency channel estimator for MC-CDMA systems with antenna arrays in frequency-selective fading environment," in Proc. VTC'01, vol. 4, 2001, pp. 2173-2177.

[15] T. M. Lok, T. F. Wong, and J. S. Lehnert, "Blind adaptive signal reception for MC-CDMA systems in Rayleigh fading channels," IEEE Trans. Commun., vol. 47, pp. 464-471, Mar. 1999.

[16] Y. Sanada, M. Padilla, and K. Araki, "Performance of adaptive array antennas with multicarrier DS/CDMA in a mobile fading environment," IEICE Trans. Commun., vol. E81-B, pp. 1392-1400, July 1998.

[17] S. C. Park and J. F. Doherty, "Generalized projection algorithm for blind interference suppression in DS/CDMA communications," IEEE Trans. Circuits Syst. II, vol. 44, pp. 453-460, June 1997.

[18] J. von Neumann, Functional Operators. Princeton, NJ: Princeton Univ. Press, 1950, vol. II, Annals of Mathematical Studies.

[19] H. Stark and Y. Yang, Vector Space Projections, 1st ed. New York: Wiley, 1998.

[20] A. Klein, G. K. Kaleh, and P. W. Baier, "Zero forcing and minimum mean-square-error equalization for multiuser detection in code-division multiple-access channels," IEEE Trans. Veh. Technol., vol. 45, pp. 276-297, May 1996.

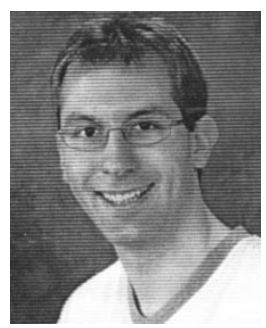

David J. Sadler (M'01) received the M.Eng. degree in engineering science from the University of Oxford, Oxford, U.K., in 1998. In 2000, he joined the Communications and Signal Processing Research Group at Imperial College London, London, U.K., to pursue the Ph.D. degree.

Since 1998, he has been employed by Roke Manor Research Ltd., Hampshire, U.K., as a member of the Array Technology skill group where he has worked on a variety of projects concerned with sensing and communications systems. His primary research interests are in the area of mobile communication systems, particularly those using antenna arrays for capacity and service enhancement. His other interests include multicarrier air interfaces, and multiuser detection and channel estimation techniques.

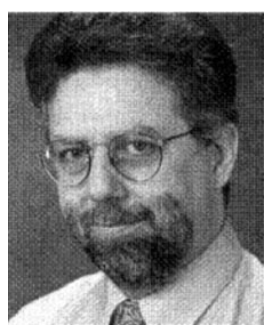

Athanassios Manikas (M'88) was appointed as a Lecturer at Imperial College London in 1988 and is now a Reader in Digital Communications in the Department of Electrical and Electronic Engineering (http://skynet.ee.imperial.ac.uk/manikas.html). $\mathrm{He}$ has published an extensive set of journal and conference papers relating to his research work, which is in the general area of digital communication and signal processing, where he has developed a wide and deep interest in the topic of superresolution arrays. His current research work on Array Processing and Array Communications is supported by the Engineering and Physical Sciences Research Council (EPSRC) U.K. as well as by the Defence Technology Centre (DTC) U.K. Dr Manikas has had various technical chairs in international conferences while he is currently the Deputy Head of the Communications and Signal Processing Research Group at Imperial College London.

Dr. Manikas is a fellow of the Institution of Electrical Engineers (IEE). 